\title{
Article \\ Mouse Gastric Epithelial Cells Resist CagA Delivery by the Helicobacter pylori Type IV Secretion System
}

\author{
Rejina Shrestha ${ }^{1}$, Naoko Murata-Kamiya ${ }^{1}$, Satoshi Imai ${ }^{1}\left(\mathbb{D}\right.$, Masami Yamamoto $^{2}$, Tetsuya Tsukamoto ${ }^{3}(\mathbb{D}$, \\ Sachiyo Nomura ${ }^{4}$ iD and Masanori Hatakeyama ${ }^{1, *}$ (D)
}

1 Division of Microbiology, Graduate School of Medicine, The University of Tokyo, 7-3-1 Hongo, Bunkyo-ku, Tokyo 113-0033, Japan; rejinasth6@gmail.com (R.S.); naokokam@m.u-tokyo.ac.jp (N.M.-K.); isatoshi@m.u-tokyo.ac.jp (S.I.)

2 Division of Physiological Pathology, Department of Applied Science, School of Veterinary Nursing and Technology, Nippon Veterinary and Life Science University, 1-7-1 Kyonan-cho, Musashino-shi, Tokyo 180-8602, Japan; masami@nvlu.ac.jp

3 Department of Diagnostic Pathology, Fujita Health University School of Medicine, 1-98 Dengakugakubo, Kutsukake-cho, Toyoake 470-1192, Japan; ttsukamt@fujita-hu.ac.jp

4 Department of Gastrointestinal Surgery, Graduate School of Medicine, The University of Tokyo, 7-3-1 Hongo, Bunkyo-ku, Tokyo 113-0033, Japan; snomura-gi@umin.ac.jp

* Correspondence: mhata@m.u-tokyo.ac.jp; Tel.: +81-3-5841-3404

\section{check for}

updates

Citation: Shrestha, R.;

Murata-Kamiya, N.; Imai, S.;

Yamamoto, M.; Tsukamoto, T.;

Nomura, S.; Hatakeyama, M. Mouse Gastric Epithelial Cells Resist CagA Delivery by the Helicobacter pylori

Type IV Secretion System. Int. J. Mol. Sci. 2022, 23, 2492. https://doi.org/ $10.3390 /$ ijms 23052492

Academic Editor: Francesco

Boccellato

Received: 20 December 2021

Accepted: 21 February 2022

Published: 24 February 2022

Publisher's Note: MDPI stays neutral with regard to jurisdictional claims in published maps and institutional affiliations.

Copyright: () 2022 by the authors. Licensee MDPI, Basel, Switzerland. This article is an open access article distributed under the terms and conditions of the Creative Commons Attribution (CC BY) license (https:/ / creativecommons.org/licenses/by/ $4.0 /)$.

\begin{abstract}
The initial step in bacterial infection is adherence of the bacterium to the target cell surface. Helicobacter pylori exploits the interaction of bacterial adhesin protein HopQ with human epithelial CEACAMs (CEACAM1, 5, and 6) to stably adhere to gastric epithelial cells, which is necessary for delivery of the H. pylori CagA oncoprotein into the epithelial cells via a type IV secretion system. In contrast to human CEACAMs, however, HopQ does not interact with Ceacam1 (mouse CEACAM1) in vitro or in $\mathrm{CHO}$ cells ectopically expressing Ceacam1. Since the mouse genome lacks Ceacam5 and Ceacam6, no significant HopQ-Ceacam interaction may occur in mouse gastric epithelial cells. Here, we found that the mouse stomach has a much lower expression level of Ceacam 1 than the expression level of CEACAM1 in the human stomach. Consistently, mouse gastric epithelial cells resist CagA delivery by cagA-positive H. pylori, and the delivery is restored by ectopic expression of human CEACAM1 or CEACAM5 in mouse gastric epithelial cells. Thus, despite the fact that mice are routinely used for $H$. pylori infection studies, a low expression level of Ceacam 1 in the mouse stomach together with the loss or greatly reduced interaction of HopQ with Ceacams make the mouse an inappropriate model for studying the role of H. pylori-delivered CagA in gastric pathogenesis, including the development of gastric cancer.
\end{abstract}

Keywords: Helicobacter pylori; mouse gastric epithelial cells; CEACAM; CagA; HopQ

\section{Introduction}

Chronic infection with Helicobacter pylori plays an etiologic role in most if not all human gastric cancers, the third leading cause of cancer-related deaths worldwide, making the gastric pathogen the strongest risk factor for the development of gastric cancer $[1,2]$. $H$. pylori is classified into two major subgroups depending on the presence or absence of the $\operatorname{cag} A$ gene, which is located on the cag pathogenicity island (cagPAI), an approximately $40 \mathrm{kbp}$ DNA segment that encodes the type IV secretion system (T4SS) [3], the bacterial microsyringe through which the $\operatorname{cag} A$-encoded CagA protein is delivered into the attached gastric epithelial cells [4-6]. Infection with $\operatorname{cag} A$-positive strains has been considered to be responsible for the development of gastric cancer at least in part by injecting CagA into gastric epithelial cells [7-9]. Once delivered inside the host cells, CagA is tethered to the inner plasma membrane where it acts as a protein scaffold that promiscuously interacts with multiple host proteins such as the oncogenic phosphatase SHP2 and polarityregulating kinase PAR1b and perturbs their physiological functions, thereby contributing to 
the neoplastic transformation of CagA-delivered gastric epithelial cells [10-16]. Consistent with this, transgenic expression of CagA in mice gave rise to spontaneous gastrointestinal and hematological malignancies, confirming the oncogenic potential of the bacterial CagA protein in mammals [17].

The attachment of $H$. pylori to the human gastric epithelial cell surface involves BabA and SabA adhesins, which are outer membrane proteins (OMPs) that bind to Lewis $b$ (Leb) and sialyl-Lewis x (sLex) antigens, respectively [18,19]. H. pylori-mediated CagA translocation is initiated by the interaction of CagA with host membrane phosphatidylserine and is facilitated by $\beta 1$ integrin interaction of cagPAI-encoded T4SS components including CagL, CagA, CagY, and CagI [20,21]. Furthermore, recent studies have revealed that the H. pylori HopQ adhesin protein interacts with the amino-terminal immunoglobulin-like domain of human carcinoembryonic antigen-related cell adhesion molecules (CEACAMs) with high affinity [22,23], and this strong interaction is indispensable for H. pylori-mediated CagA delivery.

Human CEACAMs comprise 12 members of immunoglobulin-related glycoproteins. Some members such as CEACAM1, CEACAM3, and CEACAM4 have a transmembrane domain followed by a cytoplasmic domain and others such as CEACAM5, CEACAM6, CEACAM7 and CEACAM8 are anchored to the cell membrane via a glycosylphosphatidylinositol (GPI) linkage. CEACAMs display different expression patterns on different cell types and mediate multiple cellular processes including cell adhesion, differentiation, proliferation, motility, survival, and immune responses, through homophilic and/or heterophilic interactions with other molecules to transmit signals into the cells [24-26]. Several CEACAMs also serve as adhesions for bacteria and/or viruses, playing an important role in the clearance of microbial pathogens by innate immunity [24]. Gastric epithelial cells express the transmembrane receptor CEACAM1 as well as GPI-anchored CEACAM5 and CEACAM6 [22,23]. Notably, the CEACAM family has expanded considerably during primate evolution; only a few CEACAM orthologues are present in rodents and rodents do not possess GPI-anchored membrane Ceacams (mouse CEACAM) [25-27]. H. pylori HopQ strongly binds to the N-terminal IgV-like domain of human CEACAM1, 3, 5, and 6 with high affinity in vitro $\left(K_{\mathrm{D}} \sim 100 \mathrm{nM}\right)$, but not CEACAM8, and among these, CEACAM1, 5, and 6 are expressed in various tissues/organs including the stomach epithelium (hereafter denoted here as epithelial CEACAMs) [22,23], while CEACAM3 and 8 are specifically expressed in hematopoietic cells [26]. The binding of HopQ to CEACAMs is crucial for H. pylori-mediated CagA delivery into human gastric epithelial cells, indicating that CEACAM1, 5, and 6 are involved in the process. Furthermore, the HopQ-CEACAM interaction is species-specific, since HopQ did not significantly bind to Ceacam1 (mouse CEACAM1) [22,23].

Although mice are not natural $H$. pylori hosts, mice have been used in many studies for artificial infection with $H$. pylori cagA-positive strains to study $H$. pylori-mediated gastric diseases including gastric cancer. Although infection of mice with $\operatorname{cag} A$-positive strains gave rise to more severe mucosal damage than infection with $c a g A$-negative strains did, there has been no report of induction of gastric cancer in mice with long-term cagA-positive H. pylori infection. In contrast, transgenic expression of CagA in mice gave rise to the spontaneous development of gastrointestinal and hematological malignancies [17]. These findings raise the possibility that cagA-positive $H$. pylori cannot efficiently deliver CagA into mouse gastric epithelial cells due to lack of Ceacams that bind H. pylori HopQ. In the present study, we examined the ability of cagA-positive H. pylori to deliver CagA into mouse gastric epithelial cells in the context of HopQ-Ceacam interactions.

\section{Results}

2.1. CagA-Positive Helicobacter pylori Is Capable of Delivering CagA into Human Gastric Epithelial Cells but Not Mouse Gastric Epithelial Cells

Quantitative analysis of mRNA expression for epithelial CEACAMs/Ceacams in the stomach using a public database [NCBI Gene, https://www.ncbi.nlm.nih.gov/gene/] 
(accessed on 15 December 2021) revealed that the expression of mouse Ceacam1 was 5-to-10fold less than that of human CEACAM1 (Figure 1) [28,29]. The absence of genes encoding GPI-anchored Ceacams in the mouse genome $[25,27]$ indicates that there is no expression of mouse orthologues of human CEACAM5 and CEACAM6 in the mouse stomach. Whereas Ceacam 1 has a duplicated homologue Ceacam 2 in the mouse genome, its mRNA was detected at a very low level in the alimentary tract (Figure 1). Thus, Ceacam1 may be almost all of the epithelial Ceacams expressed in mouse gastric epithelial cells. Together with the results of previous studies showing that $H$. pylori HopQ does not significantly bind to mouse Ceacam1 $[22,23]$ and the fact that mice do not possess GPI-anchored membrane Ceacams such as those corresponding to human CEACAM5 and CEACAM6, the finding raises the possibility that $\mathrm{H}$. pylori interacts much less efficiently with mouse gastric epithelial cells than with human epithelial cells because of the low expression level of Ceacam1 with markedly reduced HopQ-binding activity, which dampens H. pylori T4SS-mediated CagA delivery.

A

mRNA expression in human stomach

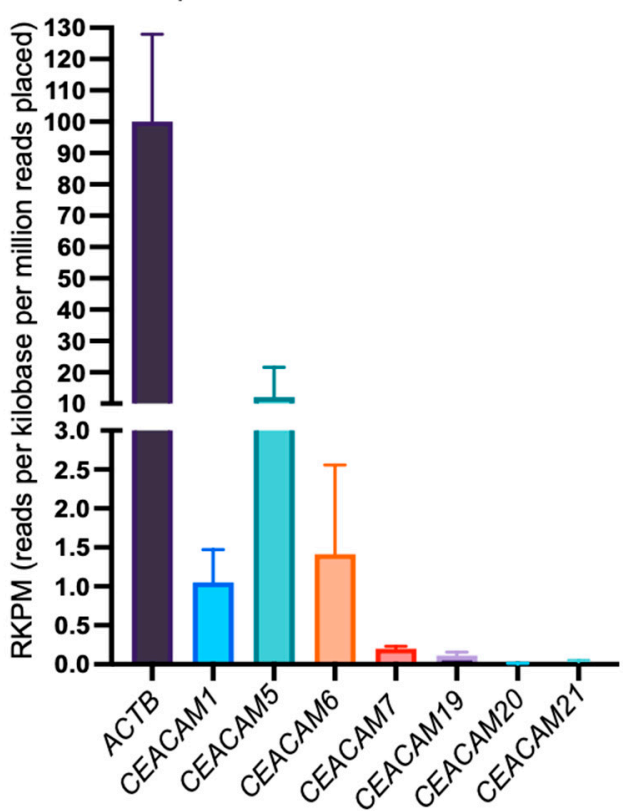

B

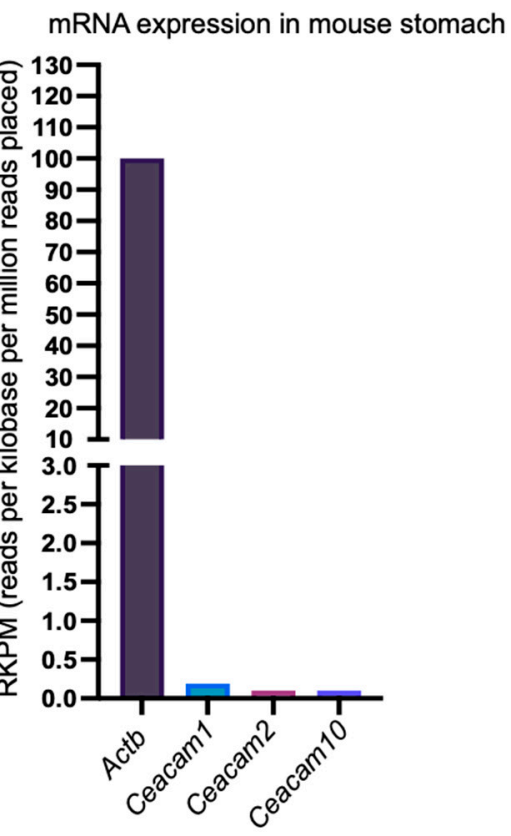

Figure 1. Relative expression levels of human and mouse mRNAs encoding membrane CEACAMs (human) and membrane Ceacams (mouse) in the stomach. Relative levels of human CEACAM1, CEACAM5, CEACAM6, CEACAM7, CEACAM19, CEACAM20, and CEACAM21 mRNA expression compared to $A C T B$ ( $\beta$-ACTIN mRNA) in human stomach (A). Relative levels of mouse Ceacam1, Ceacam2, and Ceacam10 mRNA compared to Actb ( $\beta$-Actin) mRNA in mouse stomach (B). It should be noted that the mouse genome does not contain human CEACAM5, CEACAM6, and CEACAM7 orthologues, whereas the human genome does not contain a mouse Ceacam 2 orthologue.

To test the above-described possibility, we used four mouse gastric epithelial cell lines-YTN2, YTN3, YTN5 and YTN16 - that were established from gastric carcinoma induced by treating C57BL/ 6 mice with $N$-methyl- $N$-nitrosourea (MNU) [30]. Consistent with the mRNA expression profile in the stomach (Figure 1), immunoblotting analysis using an anti-CEACAM1 antibody, which recognizes both human CEACAM1 and mouse Ceacam1, revealed that the expression level of Ceacam1 in mouse gastric epithelial cells was much lower than the expression level of CEACAM1 in AGS human gastric cancerderived epithelial cells (Supplementary Figure S1). We next conducted an in vitro infection experiment using gastric epithelial cells infected with the $H$. pylori cagA-positive Western standard strain NCTC11637 strain. (Hereafter "H. pylori" denotes the NCTC11637 cagA 
positive strain unless otherwise stated.) Since delivered CagA undergoes tyrosine phosphorylation by host cell kinases [31,32], the level of tyrosine-phosphorylated CagA represents the amounts of CagA delivered into gastric epithelial cells by H. pylori. As in many other studies, AGS cells were used as positive control cells for the infection experiment. Since a CagA delivery experiment is usually performed by incubation of gastric cells with $H$. pylori for 3-7 h, during which the amount of delivered CagA is proportional to the duration of incubation, we used $7 \mathrm{~h}$ incubation to see maximal CagA delivery by the bacterial infection. At $7 \mathrm{~h}$ after the onset of $H$. pylori infection, cell lysates were prepared from infected gastric epithelial cells and subjected to immunoblotting with an anti-CagA antibody that detects both CagA derived from host cell surface-attached H. pylori as well as CagA delivered into host cells and with an anti-phosphotyrosine (pTyr) antibody that specifically detects host cell-delivered CagA. The results of the experiment showed that a substantial amount of H. pylori was attached to the surface of human gastric epithelial cells, to which CagA was efficiently delivered into the cells, as determined by the tyrosine-phosphorylated CagA band. In striking contrast, only a small amount of $H$. pylori was attached to and only a small amount of CagA was delivered into mouse gastric epithelial cells (Figure 2). The same results were reproducibly obtained when another H. pylori cagA-positive strain (G27) was used for the infection experiment (Supplementary Figure S2). The results indicated that, in contrast to the case of human gastric epithelial cells, $H$. pylori neither associated stably with mouse gastric epithelial cells nor efficiently delivered CagA into mouse gastric epithelial cells.

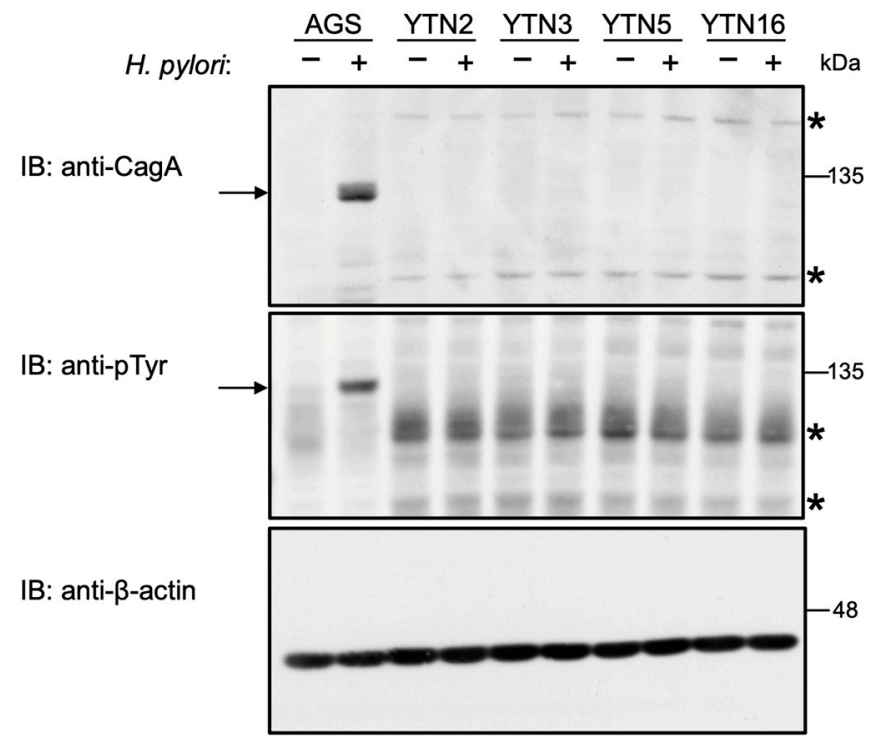

Figure 2. Infection with cagA-positive $H$. pylori is not able to deliver CagA into mouse gastric epithelial cells. Mouse gastric epithelial cells (YTN2, YTN3, YTN5, and YTN16) and AGS human gastric epithelial cells were infected with $H$. pylori NCTC11637 strain for $7 \mathrm{~h}$ at a MOI of 100 . The cells were harvested and analyzed for CagA and tyrosine phosphorylation (pTyr) of CagA proteins. CagA is detectable at $135 \mathrm{kDa}$ as indicated by the arrow in the anti-CagA and anti-pTyr immunoblot. Asterisks $\left({ }^{*}\right)$ denote the non-specific band observed for cell lysates of mouse origins. Representative image of two independent experiments. Experiments were carried out in biological duplicates $(n=2)$ and the representative images are shown.

\subsection{The CagA Protein Is Efficiently Translocated and Phosphorylated in Human CEACAM- Expressing Mouse Gastric Epithelial Cells}

To examine whether impairment of CagA delivery into mouse gastric epithelial cells by $H$. pylori was due to the weak interaction of $H$. pylori HopQ with mouse Ceacams both qualitatively and quantitatively, we established transfectant cells that stably express hemagglutinin (HA)-tagged human CEACAM1 (the CEACAM1-4s isoform), human CEACAM5, 
or human CEACAM6 using two mouse gastric epithelial cell lines-YTN2 and YTN16which were arbitrarily chosen from the four mouse gastric epithelial cell lines as they displayed similar morphology and growth rates (Figure 3). The broad bands corresponding to human CEACAM1, 5, and 6 shown in immunoblotting were consistent with the notion that they were heavily glycosylated by post-translational modification.

A

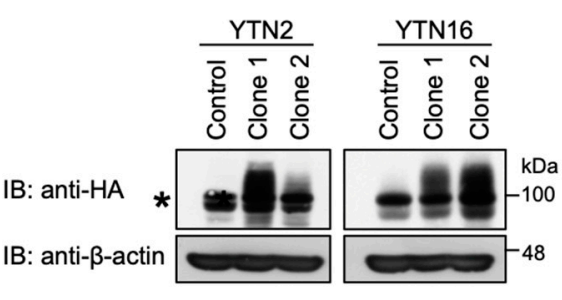

C Human CEACAM 6

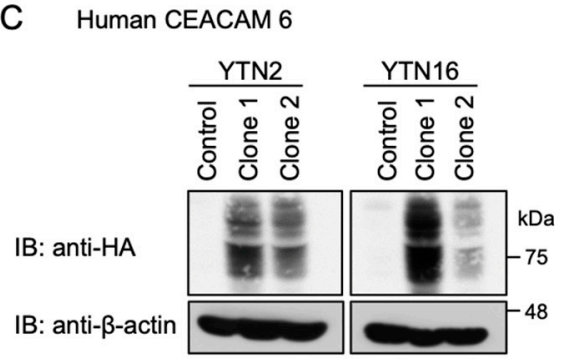

B Human CEACAM5

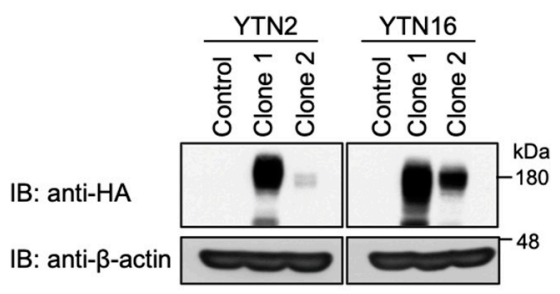

Figure 3. Establishment of human CEACAM-expressing mouse gastric epithelial cells. (A) YTN2and YTN16-derived hemagglutinin (HA)-tagged human CEACAM1 stable cells (clone 1 and clone 2) were harvested and analyzed for expression. Parental cell lines were used as control. The asterisk $\left(^{*}\right)$ denotes the non-specific band observed by immunoblotting of YTN2 and YTN16 mouse cell lysates with a human CEACAM1 antibody. (B) YTN2- and YTN16-derived HA-tagged human CEACAM5 stable cells (clone 1 and clone 2) were harvested and analyzed for expression. Parental cell lines were used as control. (C) YTN2- and YTN16-derived HA-tagged human CEACAM6 stable cells (clone 1 and clone 2) were harvested and analyzed for expression. Parental cell lines were used as control. CEACAMs are recognized as broad bands as they are heavily glycosylated in the cells.

The YTN2 or YTN16 cell-derived transfectants stably expressing human CEACAM1 were then infected with H. pylori. Ectopic expression of human CEACAM1 in mouse gastric epithelial cells made it possible for $\operatorname{cag} A$-positive $H$. pylori to deliver CagA into the cells as determined by tyrosine-phosphorylated CagA (Figure 4A, Supplementary Figure S3). Likewise, stable expression of human CEACAM5, which does not have a mouse orthologue, enabled CagA delivery into mouse gastric epithelial cells by cagA-positive H. pylori infection (Figure 4A, Supplementary Figure S4). In the infection study, we detected a larger amount of CagA proteins in lysates prepared from YTN2-derived cells stably expressing CEACAM1 (YTN2/hCEACAM1) or CEACAM5 (YTN2/hCEACAM5), possibly due to increased binding of $H$. pylori to the surface of mouse gastric epithelial cells expressing these human CEACAM proteins (Figure 4A, Supplementary Figures S3 and S4), providing additional evidence that both human CEACAM1 and human CEACAM5 efficiently bind to H. pylori HopQ. Since H. pylori HopQ also interacts with human CEACAM6 in vitro [22,23], we also generated stable human CEACAM6 transfectants from YTN2 or YTN16 cells as parental cells and infected them with $H$. pylori for $7 \mathrm{~h}$. Despite sufficient expression of human CEACAM6 on the stable transfectants, there was no evidence for CagA delivery (Figure 4A, Supplementary Figure S5). From these observations, we concluded that $H$. pylori CagA can be translocated and tyrosine-phosphorylated into mouse gastric epithelial cells stably expressing human CEACAM1 or human CEACAM5 but not into cells stably expressing human CEACAM6. In this regard, it should be noted that the AGS cell lysates used for positive controls (third panel from the top) did not display bands corresponding 
to human CEACAM1, 5, and 6. This was simply because the immunoblotting experiment was carried out by using an anti-HA antibody that detects ectopically expressed human CEACAMs but not endogenous human CEACAMs. Indeed, stripping and reprobing of the same filter with an antihuman CEACAM1 antibody revealed the presence of endogenous CEACAM1 in AGS cells (Figure 4B).

A

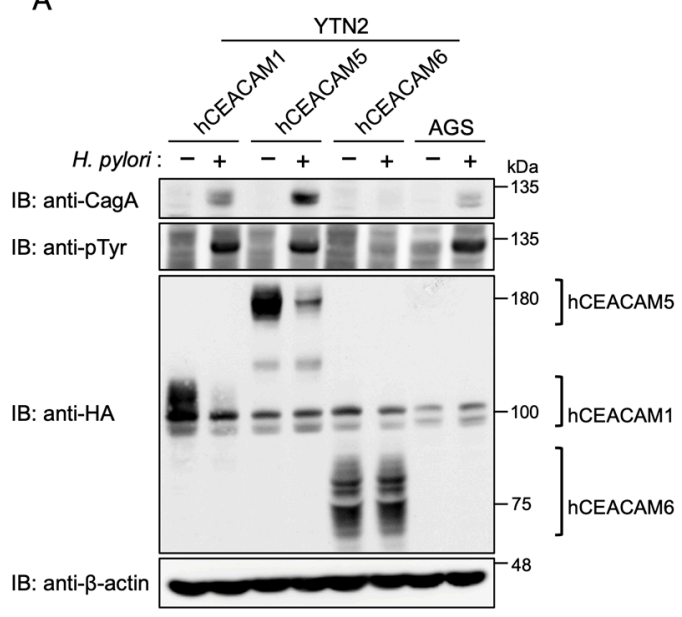

B

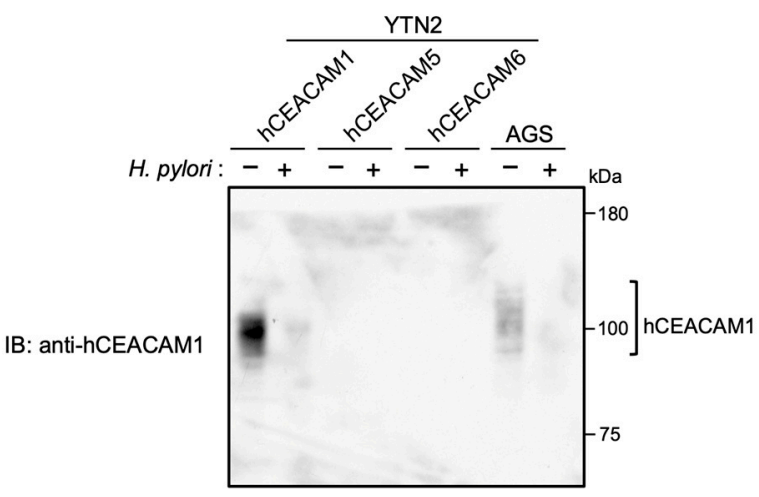

Figure 4. Infection with cagA-positive H. pylori is capable of delivering CagA into mouse gastric epithelial cells expressing human CEACAM. YTN2 cell-derived hemagglutinin (HA)-tagged human CEACAM1 (hCEACAM1), human CECAM5 (hCEACAM5), and human CEACAM6 (hCEACAM6) stable cells and AGS cells were infected with H. pylori NCTC11637 for $7 \mathrm{~h}$ at a MOI of 100. The cells were harvested and analyzed for CagA, tyrosine phosphorylation (pTyr) of CagA, and HA-tagged CEACAM proteins. The broad bands represent heavily glycosylated bands of CEACAMs (A). The anti-HA membrane was reprobed with an antihuman CEACAM1 (B). Experiments were carried out in biological duplicates $(n=2)$ and the representative images are shown. See also Supplementary Figures S3-S5.

\subsection{HopQ Interaction Transiently Reduces the Cellular Levels of Cellular CEACAM Protein}

During the course of the H. pylori infection experiment, we noticed that the level of ectopically expressed human CEACAM1 or CEACAM5 was substantially diminished after $7 \mathrm{~h}$ of infection with wild-type $H$. pylori (Figure 4A, indicated by comparison of lane 1 and lane 2 for human CEACAM1 and by comparison of lane 3 and lane 4 for human CEACAM5). Along with ectopic expression, endogenous CEACAM1 expression was also reduced (Figure 4B; lane 7 and lane 8). In contrast, there was no decrease in the level of human CEACAM6 at $7 \mathrm{~h}$ after cagA-positive $H$. pylori infection (Figure $4 \mathrm{~A}$, indicated by comparison of lane 5 and lane 6). Hence, the reduction in human CEACAM was correlated with its ability to bind to H. pylori HopQ and subsequent delivery of CagA into host cells. Given this, we next investigated the relationship between the functional T4SS and reduction in human CEACAM1 expression. For this purpose, we infected AGS cells with wild-type H. pylori and its isogenic $\Delta$ virD4 strain that lack the functional T4SS (Figure 5). The results of the experiment revealed that tyrosine phosphorylation of CagA was detectable within $1 \mathrm{~h}$ after wild-type $H$. pylori infection. Reduced CEACAM1 expression was also detected within $1 \mathrm{~h}$ after infection and the magnitude of CEACAM1 reduction was correlated with elevated levels of CagA tyrosine phosphorylation in a time-dependent manner (Figure 5). In AGS cells infected with the $\triangle$ virD4 isogenic strain, the level of CEACAM1 expression was stable and constitutive during $7 \mathrm{~h}$ infection. Thus, a reduction in human CEACAM1 expression was substantially dependent on the presence of the functional T4SS (Figure 5). 


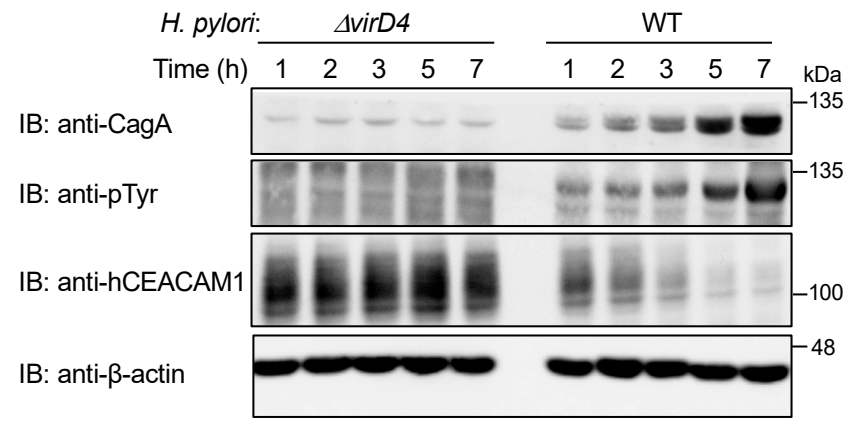

Figure 5. H. pylori T4SS contributes to deduction of the level of cellular CEACAM protein. AGS cells were infected with wild-type (WT) H. pylori or isogenic $\Delta v i r D 4$ strain for consecutively $7 \mathrm{~h}$ at a MOI of 100. The cells were harvested and analyzed for CagA, tyrosine phosphorylation (pTyr) of CagA, and human CEACAM1 (hCEACAM1) proteins. Experiments were carried out in biological duplicates $(n=2)$ and the representative images are shown.

We then investigated the possibility that CagA delivered into gastric epithelial cells via the functional T4SS generates a signal that downregulates CEACAM1 or CEACAM5 expression. To test this idea, we infected AGS cells with wild-type H. pylori or its isogenic strain lacking the $\operatorname{cag} A$ gene $(\triangle \operatorname{cag} A)$. The results of the experiment revealed a slight increase in the level of human CEACAM1 expression upon infection with the $\triangle$ cag $A$ strain (Figure 6). Similar results were observed in Kato-III cells (Supplementary Figure S6). Reciprocally, ectopic expression of cDNA-delivered CagA in AGS cells gave a slight reduction in the level of human CEACAM1 (Figure 7). These results indicated that the functional T4SS plays a major role in the reduction in CEACAM1 and CEACAM5 by H. pylori infection, although the contribution of delivered CagA was marginal if any. In addition, to evaluate the time span of reduction in CEACAM1 expression upon H. pylori infection, AGS cells and Kato-III cells were infected with cagA-positive $H$. pylori for time frames of 0,7 , and $24 \mathrm{~h}$, and CEACAM1 expression was evaluated in the above-mentioned time frames of infection. It was observed that the expression level of CEACAM1 decreased at $7 \mathrm{~h}$ as observed previously, which was restored by $24 \mathrm{~h}$ of infection (Supplementary Figure S7), suggesting that the expression of CEACAM1 oscillates throughout the infection period.
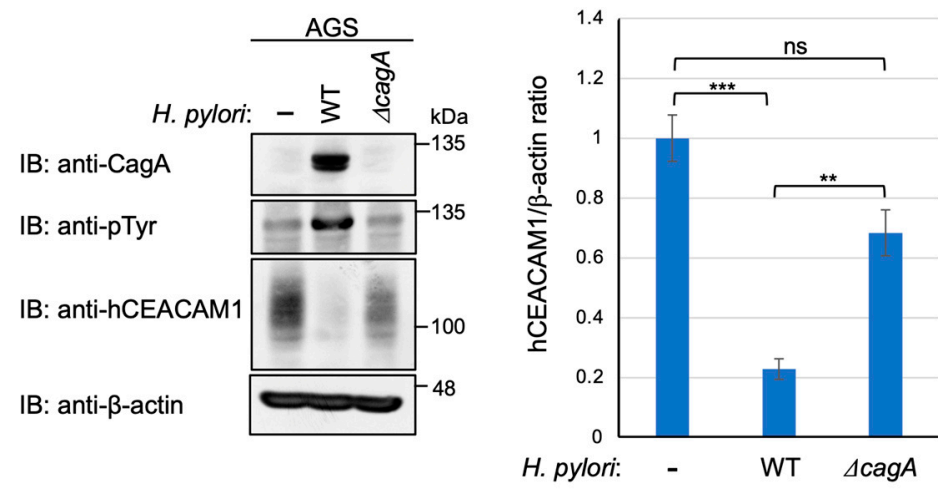

Figure 6. CEACAM-mediated CagA delivery reduces the level of cellular CEACAM protein. AGS cells were infected with wild-type (WT) H. pylori or isogenic $\Delta$ cagA strain for $7 \mathrm{~h}$ at a MOI of 100 . The cells were harvested and analyzed for the expression of total CEACAM1 protein. Experiments were carried out in biological triplicates $(n=3)$ and the representative images are shown (left). Quantification of the intensity of CEACAM1 relative to $\beta$-actin (right). Error bars indicate mean \pm SD, $n=3$. Data were analyzed by one-way ANOVA and the Bonferroni post hoc test. ${ }^{* * *} p<0.001$, ** $p<0.01$, ns; non-significant. 


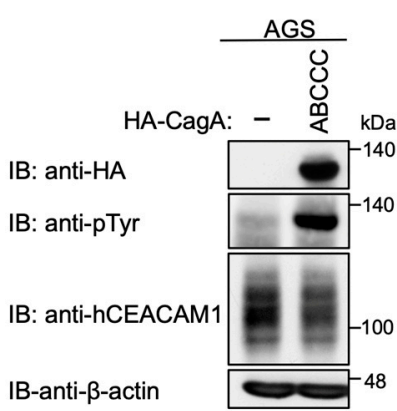

Figure 7. Plasmid-mediated expression of CagA is only a modest reduction in the level of cellular CEACAM protein. AGS cells were transfected with HA-tagged CagA expression vector. The cell lysates were subjected to immunoblotting with indicated antibodies. Experiments were carried out in biological triplicates $(n=3)$ and the representative images are shown (left). Quantification of the intensity of CEACAM1 relative to $\beta$-actin (right). Error bars indicate mean $\pm \mathrm{SD}, n=3 .{ }^{*} p<0.05$.

\section{Discussion}

We found in the present study that, in stark contrast to human gastric epithelial cells, mouse gastric epithelial cells resist T4SS-mediated CagA injection by H. pylori cagApositive strains. Though not natural hosts of $H$. pylori, mice have been routinely used for in vivo studies of $H$. pylori infection. However, in most cases, H. pylori-infected mouse only develop lymphocytic gastritis without progression to severe gastric lesions including gastric cancer [33-35]. Only a few cagPAI-positive strains have been successfully adapted for long-term colonization in the mice stomach and infection of mice with these strains can lead to the development of chronic active gastritis with neutrophil infiltration, progressing to atrophy and metaplasia [36,37]. Nevertheless, even long-term infection of mice with such $\operatorname{cag} A$-positive strains does not spontaneously induce gastric cancer, indicating that mice do not faithfully phenocopy gastric lesions induced by chronic cagA-positive H. pylori infection in human. Since transgenic expression of CagA in mouse gastric epithelial cells gave rise to the induction of gastric cancer [17,38], the failure of gastric cancer development in the stomach of mice infected with cagA-positive $H$. pylori does not seem to be due to the insensitivity of mouse gastric epithelial cells to the oncogenic action of CagA. Instead, the results of the present study revealed that $c a g A$-positive $H$. pylori failed and is incapable of delivering the CagA oncoprotein into mouse gastric epithelial cells.

It has been shown that the $H$. pylori adhesin HopQ is capable of strongly interacting with several CEACAM proteins including CEACAM1, CEACAM3, CEACAM5, and CEACAM6 and that the HopQ-CEACAM interaction is crucial for T4SS-mediated delivery of CagA by H. pylori. Since human CEACAM3 is specifically expressed on granulocytes [26], it does not seem to play a substantial role in the delivery of CagA into gastric epithelial cells. Previous studies also showed that the HopQ-binding ability of mouse Ceacam1 is much less than that of human CEACAM1 (if any) [22,23]. In the present study, we found that the mouse stomach expresses only a small amount of Ceacam1. Although mice possess Ceacam2, a Ceacam 1 homologue ( $80 \%$ similarity) that is absent in humans, Ceacam 2 is expressed at a very low level in the mouse stomach [29]. Together with the fact that the mouse genome does not possess Ceacam 5 and Ceacam6 genes [24,25], the present study revealed that epithelial cells in the mouse stomach do not express Ceacams that are capable of strongly binding with $H$. pylori HopQ both qualitatively and quantitatively. As a consequence, while cagA-positive $H$. pylori binds to the surface of mouse gastric epithelial cells via adhesins such as BabA, SabA, and LabA, their weak interactions do not allow substantial CagA delivery into mouse gastric epithelial cells via the T4SS in the absence of HopQ-Ceacam interaction (Figure 8). 


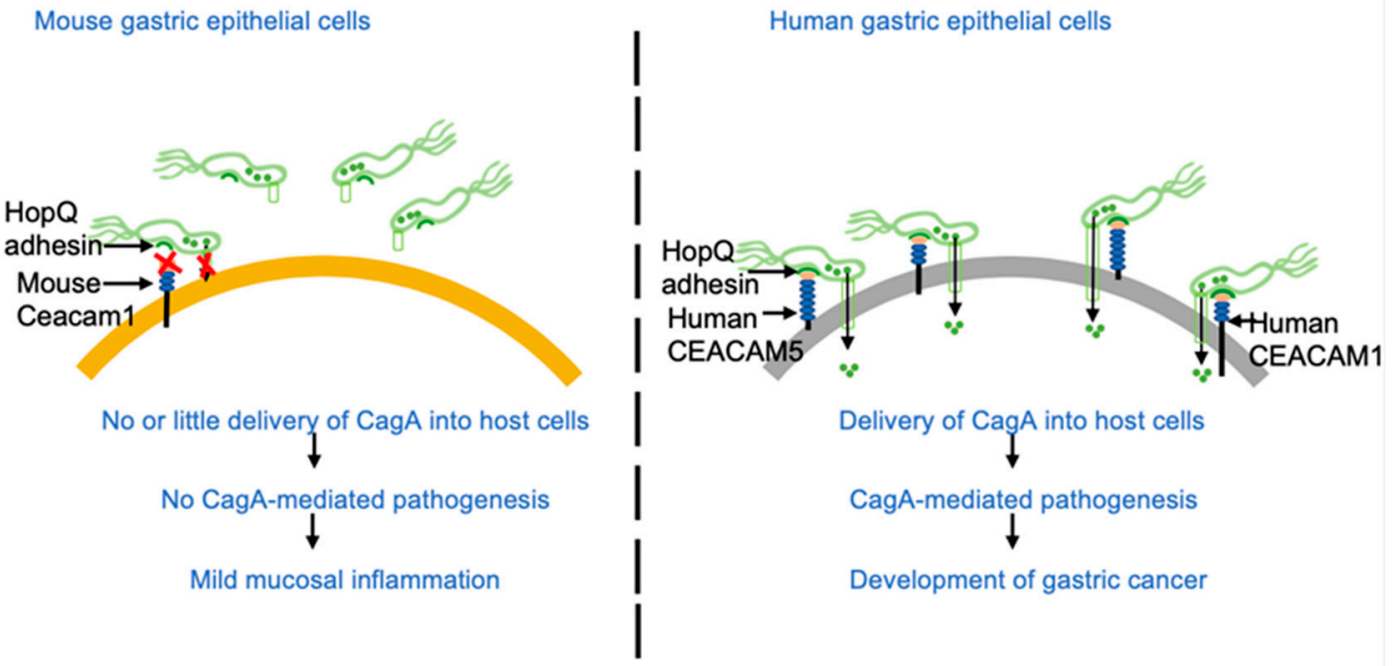

Figure 8. Schematic representation of H. pylori infection in mouse and human gastric epithelial cells. Due to low expression of mouse Ceacam 1 followed by the incompatible interaction between mouse Ceacam1 and H. pylori HopQ, there is no or little delivery of CagA in the mouse gastric epithelial cells. In contrast, compatible binding of H. pylori HopQ to expressed human CEACAMs leads to efficient delivery of CagA in human gastric epithelial cells leading to CagA mediated pathogenesis.

A previous study showed that $H$. pylori binds to the membrane surface of Chinese hamster ovary $(\mathrm{CHO})$ cells ectopically expressing human CEACAM1 in a HopQ-dependent manner [23]. However, it was not examined in that study if CagA is delivered into human CEACAM1-expressing CHO cells. Ectopic expression of human CEACAM1, CEACAM5 or CEACAM6 in human embryonic kidney 293 (HEK293) cells reconstituted the HopQ/CEACAM interaction, which enabled translocation of H. pylori CagA into HEK293 cells [22,23]. The results of the present study also revealed that, although cagA-positive H. pylori failed to deliver CagA into mouse gastric epithelial cells, ectopic expression of a single human epithelial CEACAM such as CEACAM1 or CEACAM5 enables H. pylorimediated CagA delivery in mouse gastric epithelial cells, the bona fide targets of $H$. pylori. The results indicated that both membrane-spanning CEACAMs and GPI-anchored membrane CEACAMs are capable of provoking CagA delivery upon binding with HopQ. On the other hand, ectopic expression of another GPI-anchored CEACAM, CEACAM6, did not support $H$. pylori-mediated CagA delivery into mouse gastric epithelial cells. Since HopQ binds to recombinant CEACAM6 in vitro with high affinity [22,23], the results of the present study indicate that GPI-anchored CEACAM6 may cause an allosteric change in the IgV-like N-terminal domain that dampens the HopQ-CEACAM6 interaction. Collectively, these observations indicated that the lack of stable interaction between $H$. pylori HopQ and mouse Ceacams, which dampens CagA delivery into gastric epithelial cells, is responsible for the observed differences in pathological changes in the stomach between mice and humans infected with cagA-positive H. pylori [33-35,39].

The level of tyrosine-phosphorylated CagA, a surrogate marker of host cell-delivered CagA, in mouse gastric epithelial cells that stably express human CEACAM1 or CEACAM5 was comparable to that of CagA in AGS cells upon infection with cagA-positive H. pylori. Consistently, a substantially larger amount of CagA was detected in cell lysates prepared from gastric epithelial cells ectopically expressing human CEACAM1 or CEACAM5 than in cell lysates prepared from parental mouse gastric cells, indicating enhanced $H$. pylori adhesion to the mouse cells via ectopically expressed human CEACAM1 or CEACAM5.

Aside from the role of HopQ-CEACAM interaction in CagA delivery, we also found a time-dependent reduction in the cellular level of human CEACAM1 following $\operatorname{cag} A$ positive H. pylori infection (Figure 5). The presence of the functional T4SS but not CagA 
delivery is critical for CEACAM reduction. A reduction in human CEACAM1 in human gastric epithelial cells infected with $H$. pylori was observed for both endogenous and exogenous CEACAMs, suggesting that the downregulation occurs at the post-transcriptional level. Since the infection-associated CEACAM reduction, shown at $7 \mathrm{~h}$ after infection, was restored at $24 \mathrm{~h}$ after infection, the HopQ-CEACAM interaction provokes proteolytic cleavage of the extracellular domains of CEACAMs, which causes shedding or degradation of HopQ-bound CEACAMs. Whether the decreased HopQ-CEACAM interaction is beneficial to the host or bacterium is an important issue to be considered. The CEACAM degradation/cleavage might be advantageous for $H$. pylori as it would enable detachment of H. pylori from CagA-injected cells and movement to neighborhood epithelial cells as new targets. Alternatively, decreases in membrane-associated CEACAMs in H pylori-infected gastric epithelial cells may generate a negative feedback loop for the CagA pathogenic action of CagA, which prevents excess CagA delivery into a single epithelial cell that may provoke premature cell senescence or genomic instability depending on the functional status of p53 $[16,40]$. Molecular identification of the CEACAM proteases, which can be of human or bacterium origin, may provide a clue to understand why the H. pylori HopQhuman CEACAM1 $/ 5$ interaction undergoes transient reduction during infection of $H$. pylori carrying the functional T4SS.

The repetitive expansion of CEACAM genes in primates and rodents has been assumed to be associated with positive selection [41,42]. Previous analyses have reported CEACAM genes encompass a large number of nonsynonymous Single Nucleotide Polymorphisms (SNPs) and Copy Number Variations (CNVs) with these variations having high differentiation in the population [43]. The genetic diversity in CEACAM has been reported as a probable factor of human susceptibility to meningococcal disease [44]. The diversity in the CNVs and SNPs may also influence H. pylori binding affinity and the T4SSmediated delivery of CagA. Future investigation on the relationship between variations in CEACAMs in humans and the ability of CagA injection could explain the variability in H. pylori infection and development of gastric cancer in the infected populations.

In summary, our study has revealed the mechanisms underlying species specificity in the pathogenicity of H. pylori. To investigate the role of CagA in gastric carcinogenesis using the mouse as an in vivo model of $H$. pylori infection, it should be important to specifically express human epithelial CEACAMs in mouse gastric epithelial cells because ectopic expression of CEACAMs in multiple distinct cell types, including immune cells, might artificially modify gastric lesions induced by chronic cagA-positive $H$. pylori infection.

\section{Materials and Methods}

\subsection{Expression Vectors}

The cDNAs encoding C-terminally hemagglutinin (HA)-tagged human CEACAM 1-4s, N-terminally HA-tagged human CEACAM5 and human CEACAM6 were subcloned into a pcDNA3 vector. HA-tagged wild-type cagA derived from H. pylori NCTC11637 was cloned into the $\mathrm{pSP} 65 \mathrm{SR} \alpha$ mammalian expression vector [45].

\subsection{Cell Culture and Transfection}

AGS and Kato-III human gastric cancer cells were purchased from American Type Culture Collection (ATCC). YTN mouse gastric cancer cells were described previously [30]. AGS and Kato-III cells were cultured in RPMI 1640 containing 10\% fetal bovine serum (FBS). YTN cells (YTN2, YTN3, YTN5, and YTN16) were cultured in DMEM containing 10\% FBS and glucose $4500 \mathrm{mg} / \mathrm{L}$ using collagen type I-coated culture dishes. AGS cells and YTN16 cells were transfected with DNA using Lipofectamine 2000 reagent (Invitrogen, Carlsbad, CA, USA). YTN2 cells were transfected with DNA using Lipofectamine and PLUS reagent (Invitrogen, Carlsbad, CA, USA). 


\subsection{Helicobacter Strains and Culture}

Helicobacter pylori standard strain NCTC11637 and its isogenic strains lacking the cag $A$ gene $(\triangle c a g A)$ and lacking the virD4 gene $(\Delta v i r D 4)$ have been described previously $[45,46]$. H. pylori strains (NCTC11637 and G27) were grown in Brucella Broth supplemented with $10 \%$ FBS and incubated in a microaerobic atmosphere by using AnaeroPack helico (Mitsubishi Gas Chemical, Tokyo, Japan) at $37^{\circ} \mathrm{C}$.

\subsection{Establishment of Stable Cell Lines}

YTN2 and YTN16 cells were transfected with linearized hemagglutinin (HA)-tagged CEACAM expression vector ( $p c D N A 3-C E A C A M)$ together with linearized pBABE-puro vector. At $24 \mathrm{~h}$ post transfection, cells were incubated in culture medium containing puromycin, $8 \mu \mathrm{g} / \mathrm{mL}$ for YTN2 cells, and $12 \mu \mathrm{g} / \mathrm{mL}$ for YTN16 cells, respectively. Puromycinresistant colonies were picked up with cloning rings and then each colony was evaluated for the expression of CEACAM by immunoblotting.

\subsection{Immunoblot Analysis}

Cells were harvested and lysed in RIPA buffer $(150 \mathrm{mM} \mathrm{NaCl}, 0.1 \%$ SDS, $0.5 \%$ sodium deoxycholate, $50 \mathrm{mM}$ Tris- $\mathrm{HCl} \mathrm{pH}$ 7.5, $1 \mathrm{mM}$ EDTA, $2 \mathrm{mM} \mathrm{Na}_{3} \mathrm{VO}_{4}, 10 \mathrm{mM} \mathrm{NaF}, 10 \mathrm{mM}$ $\beta$-glycerophosphate, $2 \mathrm{mM}$ PMSF, $10 \mu \mathrm{g} / \mathrm{mL}$ leupeptin, $10 \mu \mathrm{g} / \mathrm{mL}$ trypsin inhibitor, $10 \mu \mathrm{g} / \mathrm{mL}$ aprotinin, $1 \% \mathrm{NP}-40)$. Samples were run on SDS-PAGE gel, and separated proteins were transferred to PVDF membranes (Merck Millipore, Country Cork, Ireland). After transfer, membranes were treated with a blocking solution (1\% BSA or $5 \%$ non-fat milk in $0.1 \%$ Tween $20-\mathrm{TBS})$. The membranes were incubated with primary antibodies. Anti- $\beta$-actin antibody (8H10D10), anti-hemagglutinin (HA) antibody (6E2), anti-CEACAM1 anrtibody (D3R80), and anti-CECAM1 (D1P4T) antibody were obtained from Cell Signaling Technology (Danvers, MA, USA); anti-phosphotyrosine antibody (4G10) was obtained from Millipore (Darmstadt, Germany); anti-HA antibody (3F10) was obtained from Roche (Mannheim, Germany), and anti-CagA antibody (HPP-5003-9) was obtained from Austral Biologicals (San Ramon, CA, USA). Secondary antibodies used were conjugated with horseradish peroxidase (HRP). Protein bands were visualized by Western blot chemiluminescence reagent (Perkin Elmer Life Sciences, Waltham, MA, USA).

\subsection{Statistical Analysis}

Microsoft Excel (Redmond, WA, USA) and Graph Pad Prism software (San Diego, CA, USA) were used for statistical analysis. Data were analyzed with one-way ANOVA and Bonferroni post hoc test. P values were determined by Student's t-test. All of the statistical details were stated in the figure legends. Experiments were performed in biological triplicates and representative images are displayed unless otherwise stated.

Supplementary Materials: The following are available online at https:/ / www.mdpi.com/article/10 .3390/ijms23052492/s1.

Author Contributions: Conceptualization, N.M.-K. and M.H.; methodology, N.M.-K. and S.I.; investigation, R.S. and N.M.-K.; resources, M.Y., T.T. and S.N.; writing-original draft preparation, M.H.; writing-review and editing, R.S., N.M.-K. and M.H.; supervision, M.H.; funding acquisition, M.H. All authors have read and agreed to the published version of the manuscript.

Funding: This research was supported by Japan Society for the Promotion of Science (JSPS) Grants-in Aid for Scientific Research (KAKENHI) Grant Numbers 16H06373, 16K15273, and 21H04804 (to M.H.) and 19K07535 (to N.M.-K), and by the Project for Cancer Research and Therapeutic Evolution (P-CREATE) (160200000291) from Japan Agency for Medical Research and Development (AMED) (to M.H.).

Institutional Review Board Statement: Not applicable.

Informed Consent Statement: Not applicable. 
Data Availability Statement: The data presented in this study are all contained within the main manuscript and the Supplementary Figures of this article.

Conflicts of Interest: The authors declare no conflict of interest.

\section{References}

1. Blaser, M.J.; Perez-Perez, G.I.; Kleanthous, H.; Cover, T.L.; Peek, R.M.; Chyou, P.H.; Stemmermann, G.N.; Nomura, A. Infection with Helicobacter pylori strains possessing cagA is associated with an increased risk of developing adenocarcinoma of the stomach Cancer Res. 1995, 55, 2111-2115.

2. Parkin, D.M. The global health burden of infection-associated cancers in the year. Int. J. Cancer 2002, 118, 3030-3044. [CrossRef]

3. Censini, S.; Lange, C.; Xiang, Z.; Crabtree, J.E.; Ghiara, P.; Borodovsky, M.; Rappuoli, R.; Covacci, A. cag, a pathogenicity island of Helicobacter pylori, encodes type I-specific and disease-associated virulence factors. Proc. Natl. Acad. Sci. USA 1996, 93, 14648-14653. [CrossRef]

4. Covacci, A.; Rappuoli, R. Tyrosine-phosphorylated bacterial proteins: Trojan horses for the host cell. Exp. Med. 2000, 191, 587-592. [CrossRef]

5. Backert, S.; Meyer, T.F. Type IV secretion systems and their effectors in bacterial pathogenesis. Curr. Opin. Microbiol. 2006, 9, 207-217. [CrossRef]

6. Chung, J.M.; Sheedlo, M.J.; Campbell, A.M.; Sawhney, N.; Frick-Cheng, A.E.; Lacy, D.B.; Cover, T.L.; Ohi, M.D. Structure of the Helicobacter pylori Cag type IV secretion system. eLife 2019, 8, e47644. [CrossRef]

7. Parsonnet, J.; Friedman, G.D.; Orentreich, N.; Vogelman, H. Risk for gastric cancer in people with CagA positive or CagA negative Helicobacter pylori infection. Gut 1997, 40, 297-301. [CrossRef] [PubMed]

8. Hatakeyama, M. Helicobacter pylori CagA and gastric cancer: A paradigm for hit-and-run carcinogenesis. Cell Host Microbe. 2014, 15, 306-316. [CrossRef] [PubMed]

9. Backert, S.; Blaser, M.J. The role of CagA in the gastric biology of Helicobacter pylori. Cancer Res. 2016, 76, 4028-4031. [CrossRef] [PubMed]

10. Hayashi, T.; Senda, M.; Morohashi, H.; Higashi, H.; Horio, M.; Kashiba, Y.; Nagase, L.; Sasaya, D.; Shimizu, T.; Venugopalan, N.; et al. Tertiary structure-function analysis reveals the pathogenic signaling potentiation mechanism of Helicobacter pylori oncogenic effector CagA. Cell Host Microbe 2012, 12, 20-33. [CrossRef] [PubMed]

11. Higashi, H.; Tsutsumi, R.; Muto, S.; Sugiyama, T.; Azuma, T.; Asaka, M.; Hatakeyama, M. SHP-2 tyrosine phosphatase as an intracellular target of Helicobacter pylori CagA protein. Science 2002, 295, 683-686. [CrossRef]

12. Hatakeyama, M. Oncogenic mechanisms of the Helicobacter pylori CagA protein. Nat. Rev. Cancer 2004, 4, 688-694. [CrossRef]

13. Saadat, I.; Higashi, H.; Obuse, C.; Umeda, M.; Murata-Kamiya, N.; Saito, Y.; Lu, H.; Ohnishi, N.; Azuma, T.; Suzuki, A.; et al. Helicobacter pylori CagA targets PAR1/MARK kinase to disrupt epithelial cell polarity. Nature 2007, 447, 330-333. [CrossRef]

14. Zeaiter, Z.; Cohen, D.; Müsch, A.; Bagnoli, F.; Covacci, A.; Stein, M. Analysis of detergent-resistant membranes of Helicobacter pylori infected gastric adenocarcinoma cells reveals a role for MARK2/Par1b in CagA-mediated disruption of cellular polarity. Cell. Microbiol. 2008, 10, 781-794. [CrossRef]

15. Selbach, M.; Paul, F.E.; Brandt, S.; Guye, P.; Daumke, O.; Backert, S.; Dehio, C.; Mann, M. Host cell interactome of tyrosinephosphorylated bacterial proteins. Cell Host Microbe 2009, 5, 397-403. [CrossRef]

16. Imai, S.; Ooki, T.; Murata-Kamiya, N.; Komura, D.; Tahmina, K.; Wu, W.; Takahashi-Kanemitsu, A.; Knight, C.T.; Kunita, A.; Suzuki, N.; et al. Helicobacter pylori CagA elicits BRCAness to induce genome instability that may underlie bacterial gastric carcinogenesis. Cell Host Microbe 2021, 29, 941-958. [CrossRef]

17. Ohnishi, N.; Yuasa, H.; Tanaka, S.; Sawa, H.; Miura, M.; Matsui, A.; Higashi, H.; Musashi, M.; Iwabuchi, K.; Suzuki, M.; et al. Transgenic expression of Helicobacter pylori CagA induces gastrointestinal and hematopoietic neoplasms in mouse. Proc. Natl. Acad. Sci. USA 2008, 105, 1003-1008. [CrossRef]

18. Ilver, D.; Arnqvist, A.; Ogren, J.; Frick, I.M.; Kersulyte, D.; Incecik, E.T.; Berg, D.E.; Covacci, A.; Engstrand, L.; Borén, T. Helicobacter pylori adhesin binding fucosylated histo-blood group antigens revealed by retagging. Science 1998, 279, 373-377. [CrossRef]

19. Mahdavi, J.; Sondén, B.; Hurtig, M.; Olfat, F.O.; Forsberg, L.; Roche, N.; Angstrom, J.; Larsson, T.; Teneberg, S.; Karlsson, K.A.; et al. Helicobacter pylori SabA adhesin in persistent infection and chronic inflammation. Science 2002, 297, 573-578. [CrossRef]

20. Kwok, T.; Zabler, D.; Urman, S.; Rohde, M.; Hartig, R.; Wessler, S.; Misselwitz, R.; Berger, J.; Sewald, N.; König, W.; et al. Helicobacter exploits integrin for type IV secretion and kinase activation. Nature 2007, 449, 862-866. [CrossRef]

21. Jiménez-Soto, L.F.; Kutter, S.; Sewald, X.; Ertl, C.; Weiss, E.; Kapp, U.; Rohde, M.; Pirch, T.; Jung, K.; Retta, S.F.; et al. Helicobacter pylori type IV secretion apparatus exploits $\beta 1$ integrin in a novel RGD-independent manner. PLoS Pathog. 2009, 5, e1000684. [CrossRef] [PubMed]

22. Königer, V.; Holsten, L.; Harrison, U.; Busch, B.; Loell, E.; Zhao, Q.; Bonsor, D.A.; Roth, A.; Kengmo-Tchoupa, A.; Smith, S.I.; et al. Helicobacter pylori exploits human CEACAMs via HopQ for adherence and translocation of CagA. Nat. Microbiol. 2016, 2, 16188. [CrossRef]

23. Javaheri, A.; Kruse, T.; Moonens, K.; Mejías-Luque, R.; Debraekeleer, A.; Asche, C.I.; Tegtmeyer, N.; Kalali, B.; Bach, N.C.; Sieber, S.A.; et al. Helicobacter pylori adhesin HopQ engages in a virulence-enhancing interaction with human CEACAMs. Nat. Microbiol. 2016, 2, 16189. [CrossRef] [PubMed] 
24. Kuespert, K.; Pils, S.; Hauck, C.R. CEACAMs: Their role in physiology and pathophysiology. Curr. Opin. Cell Biol. 2006, 18, 565-571. [CrossRef] [PubMed]

25. Kammerer, R.; Zimmermann, W. Coevolution of activating and inhibitory receptors within mammalian carcinoembryonic antigen families. BMC Biol. 2010, 8, 12. [CrossRef] [PubMed]

26. Tchoupa, A.K.; Schuhmacher, T.; Hauck, C.R. Signaling by epithelial members of the CEACAM family-Mucosal docking sites for pathogenic bacteria. Cell. Commun. Signal. 2014, 12, 27. [CrossRef] [PubMed]

27. Zhou, G.Q.; Zhang, Y.; Hammarström, S. The carcinoembryonic antigen (CEA) gene family in non-human primates. Gene 2001, 264, 105-112. [CrossRef]

28. Fagerberg, L.; Hallstrom, B.M.; Oksvold, P.; Kampf, C.; Djureinovic, D.; Odeberg, J.; Habuka, M.; Tahmasebpoor, S.; Danielsson, A.; Edlund, K.; et al. Analysis of the human tissue-specific expression by genome-wide integration of transcriptomics and antibody-based proteomics. Mol. Cell. Proteom. 2004, 13, 397-406. [CrossRef]

29. Yue, F.; Cheng, Y.; Breschi, A.; Vierstra, J.; Wu, W.; Ryba, T.; Sandstrom, R.; Ma, Z.; Davis, C.; Pope, B.D.; et al. A comparative encyclopedia of DNA elements in the mouse genome. Nature 2014, 515, 355-364. [CrossRef]

30. Yamamoto, M.; Nomura, S.; Hosoi, A.; Nagaoka, K.; Iino, T.; Yasuda, T.; Saito, T.; Matsushita, H.; Uchida, E.; Seto, Y.; et al. Established gastric cancer cell lines transplantable into C57BL/ 6 mice show fibroblast growth factor receptor 4 promotion of tumor growth. Cancer Sci. 2018, 109, 1480-1492. [CrossRef]

31. Stein, M.; Bagnoli, F.; Halenbeck, R.; Rappuoli, R.; Fantl, W.J.; Covacci, A. c-Src/Lyn kinases activate Helicobacter pylori CagA through tyrosine phosphorylation of the EPIYA motifs. Mol. Microbiol. 2002, 43, 971-980. [CrossRef] [PubMed]

32. Tammer, I.; Brandt, S.; Hartig, R.; König, W.; Backert, S. Activation of Abl by Helicobacter pylori: A novel kinase for CagA and crucial mediator of host cell scattering. Gastroenterology 2007, 132, 1309-1319. [CrossRef] [PubMed]

33. Sheu, B.S.; Yang, H.B.; Wu, J.J.; Huang, A.H.; Lin, X.Z.; Su, I.J. Development of Helicobacter pylori infection model in BALB/c mice with domestic cagA-positive and -negative strains in Taiwan. Dig. Dis. Sci. 1999, 44, 868-875. [CrossRef] [PubMed]

34. van Doorn, N.E.; van Rees, E.P.; Namavar, F.; de Graaff, J. Local cellular immune response in the acute phase of gastritis in mice induced chemically and by Helicobacter pylori. Med. Microbiol. 1998, 47, 863-870. [CrossRef] [PubMed]

35. Sakagami, T.; Dixon, M.; O’Rourke, J.; Howlett, R.; Alderuccio, F.; Vella, J.; Shimoyama, T.; Lee, A. Atrophic gastric changes in both Helicobacter felis and Helicobacter pylori infected mice are host dependent and separate from antral gastritis. Gut 1996, 39, 639-648. [CrossRef]

36. Franco, A.T.; Israel, D.A.; Washington, M.K.; Krishna, U.; Fox, J.G.; Rogers, A.B.; Neish, A.S.; Collier-Hyams, L.; Perez-Perez, G.I.; Hatakeyama, M.; et al. Activation of $\beta$-catenin by carcinogenic Helicobacter pylori. Proc. Natl. Acad. Sci. USA 2005, 102, 10646-10651. [CrossRef]

37. Dyer, V.; Brüggemann, H.; Sörensen, M.; Kühl, A.A.; Hoffman, K.; Brinkmann, V.; Reines, M.D.M.; Zimmerman, S.; Meyer T.F.; Koch, M. Genomic features of the Helicobacter pylori strain PMSS1 and its virulence attributes as deduced from its in vivo colonisation patterns. Mol. Microbiol. 2018, 110, 761-776. [CrossRef]

38. Miura, M.; Ohnishi, N.; Tanaka, S.; Yanagiya, K.; Hatakeyama, M. Differential oncogenic potential of geographically distinct Helicobacter pylori CagA isoforms in mice. Int. J. Cancer. 2009, 125, 2497-2504. [CrossRef]

39. Peek, R.M. Helicobacter pylori infection and disease: From humans to animal models. Dis. Model Mech. 2008, 1, 50-55. [CrossRef]

40. Saito, Y.; Murata-Kamiya, N.; Hirayama, T.; Ohba, Y.; Hatakeyama, M. Conversion of Helicobacter pylori CagA from senescence inducer to oncogenic driver through polarity-dependent regulation of p21. J. Exp. Med. 2010, 207, 2157-2174. [CrossRef]

41. Kammerer, R.; Popp, T.; Härtle, S.; Singer, B.B.; Zimmermann, W. Species-specific evolution of immune receptor tyrosine based activation motif-containing CEACAM1-related immune receptors in the dog. BMC Evol. Biol. 2007, 7, 196. [CrossRef] [PubMed]

42. McLellan, A.S.; Zimmermann, W.; Moore, T. Conservation of pregnancy-specific glycoprotein (PSG) N domains following independent expansions of the gene families in rodents and primates. BMC Evol. Biol. 2005, 5, 39. [CrossRef] [PubMed]

43. Chang, C.L.; Semyonov, J.; Cheng, P.J.; Huang, S.Y.; Park, J.I.; Tsai, H.J.; Lin, C.Y.; Grützner, F.; Soong, Y.K.; Cai, J.J.; et al. Widespread Divergence of the CEACAM/PSG Genes in Vertebrates and Humans Suggests Sensitivity to Selection. PLoS ONE 2013, 8, e61701. [CrossRef]

44. Callaghan, M.J.; Rockett, K.; Banner, C.; Haralambous, E.; Betts, H.; Faust, S.; Maiden, M.C.J.; Kroll, J.S.; Levin, M.; Kwiatkowski, D.P.; et al. Haplotypic diversity in human CEACAM genes: Effects on susceptibility to meningococcal disease. Genes Immun. 2008, 9, 30-37. [CrossRef] [PubMed]

45. Higashi, H.; Nakaya, A.; Tsutsumi, R.; Yokoyama, K.; Fujii, Y.; Ishikawa, S.; Higuchi, M.; Takahashi, A.; Kurashima, Y.; Teishikata, Y.; et al. Helicobacter pylori CagA induces Ras-independent morphogenetic response through SHP-2 recruitment and activation. J. Biol. Chem. 2004, 279, 17205-17216. [CrossRef]

46. Fujii, Y.; Murata-Kamiya, N.; Hatakeyama, M. Helicobacter pylori CagA oncoprotein interacts with SHIP2 to increase its delivery into gastric epithelial cells. Cancer Sci. 2020, 111, 1596-1606. [CrossRef] 